\title{
INDIGENISMO DE IZQUIERDA Y DE DERECHA: DOS PLANTEAMIENTOS DE LOS AÑOS 1920
}

\author{
POR \\ DAVID WISE \\ Texas Woman's University
}

Como el título sugiere, el presente trabajo versa no tanto sobre la obra literaria de José María Arguedas como sobre el debate ideológico de la década de los veinte, que hay que tener en cuenta para valorizar esa obra. Hay que señalar un notable desfase entre las sublevaciones de indígenas en la década de los diez y las tomas de posiciones políticas por parte de los intelectuales provincianos, y por sectores más amplios de la pequeña burguesía en general, en la década 1920-1930. De igual modo, existe un desfase entre esta toma de posiciones radicales, en general marxistas, y la expresión literaria de tales opciones. La obra «indigenista» de Ciro Alegría y José María Arguedas comienza realmente en 1935, y las grandes novelas El mundo es ancho y ajeno y Yawar fiesta aparecieron recién en 1941. No hay que olvidar, por supuesto, relatos precursores como El amauta Atusparia, de Ernesto Reyna, publicado en su primera edición en 1929.

También pienso que uno de los valores primordiales de las primeras obras de Arguedas - y aquí me refiero principalmente a Yawar fiestaes el de verter a forma literaria la posición radical adoptada por sectores de la intelectualidad provinciana durante los años veinte. Además, como señaló el historiador François Chevalier hace quince años, en un artículo sobre la expansión de los latifundios en el Perú a comienzos del siglo $\mathrm{xx}$, porciones de la obra de Arguedas poseen un valor documental rara vez igualado ${ }^{1}$. Chevalier se refiere al capítulo II de Yawar fiesta, «El despojo», en que Arguedas narra la usurpación de los pastos co-

${ }^{1}$ François Chevalier, «L'Expansion de la grande propriété dans le Haut-Pérou au $\mathrm{xx}^{e}$ siècle», Annales; Economies, Sociétés, Civilisations, 21, núm. 4 (París, julioagosto 1966), 815-831. 
munales y hasta de la puna puquiana por los hacendados locales, pero igualmente se podría señalar como «documento» valioso el capítulo «Los 'serranos'», que nos hace presente la fiebre de construcción vial de los años veinte, al igual que la posterior emigración de serranos a la capital y la aculturalización que allí sufren. Los hechos históricos de la formación en gran escala de clubes de provincianos y de la politicización del nuevo proletariado limeño encuentran en el capítulo «Los 'serranos'» cabal expresión.

En el presente trabajo, sin embargo, se trata principalmente de dos revistas de modesta circulación, que creo fundamentales para la investigación del fenómeno del indigenismo peruano en los años veinte. Señalan Augusta Alfajeme y Mariano Valderrama que fue en la década de los veinte cuando «la discusión de la cuestión agraria o campesina y del problema nacional se planteará con mayor profundidad y realismo y pasará a convertirse en el eje central del debate político» ${ }^{2}$. Desde diversas perspectivas de clase se publican gran cantidad de libros, revistas y ensayos que cuestionan el destino y los problemas fundamentales del Perú. Es en esta década, caracterizada por la modernización desde arriba y por la entrada en gran escala del capital norteamericano, cuando se plantean posiciones básicas respecto al futuro político del Perú.

Creo indispensable afirmar, con Tomás Escajadillo y otros, que, al hablar del período 1920-1930, se debe hablar no de «indigenismo» a secas, sino de los «indigenismos» específicos desarrollados por distintos grupos sociales, ya que el vocablo «indigenismo» era (y es) muy manoseado y la «reivindicación del indígena» constituía una consigna de mucha difusión, pero de un significado fluctuante ${ }^{3}$. Durante los años veinte habría que hablar del «indigenismo» de los mismos sectores terratenientes, del del pensamiento ilustrado de derecha, del de la pequeña burguesía radicalizada, sea de Lima o de provincias, y, finalmente, del indigenismo marxista de José Carlos Mariátegui y de los jóvenes comunistas del Cuzco. Habría que hablar, tal vez en primer lugar, del indigenismo «oficial», patrocinado por el gobierno desarrollista de Augusto B. Leguía, que subió al poder por segunda vez en 1919 y permaneció allí

${ }^{2}$ Augusta Alfajeme y Mariano Valderrama, «El surgimiento de la cuestión agraria y del llamado problema indígena», en Carlos Iván Degregori y otros, Indigenismo, clases sociales y problema nacional (Lima: Ediciones CELATS, 1978), p. 62. Este volumen se citará de aquí en adelante como Indigenismo.

${ }^{3}$ Tomás Escajadillo, «Para leer a Mariátegui», en Emilio Romero y otros, 7 ensayos: 50 años en la historia (Lima: Empresa Editora Amauta, 1979), p. 101. Véase también Alberto Flores-Galindo, «Los intelectuales y el problema nacional», en el mismo volumen, pp. 149-150. 
hasta que fue derrocado a raíz de la crisis económica del 1930. No se solidificaría el indigenismo, durante el oncenio leguiísta, en una ideología estable ni homogénea. Los intereses de clase, al par de influencias intelectuales de diversa índole, conformaron de diferentes maneras las posturas «indigenistas» adoptadas por determinados grupos. Al hablar, más adelante, del «indigenismo» manifestado en las revistas Amauta y Kúntur de un lado y de La Sierra de otro constatamos dos opciones de la pequeña burguesía intelectualizada frente a la coyuntura política de la década.

Con el segundo gobierno de Leguía, señalan Alfajeme y Valderrama -lo han señalado también muchos otros-, se produce un cambio brusco en la política oficial respecto a la población aborigen ${ }^{4}$. El Estado asume el papel de patrono de la raza indígena y otorga status jurídico a las comunidades, si bien no frena de una manera activa las usurpaciones de tierras comunales que se venían produciendo desde fines del siglo anterior y que habían alcanzado proporciones de crisis en la década 1910-1920. Leguía, apoyado por el sector progresivo de la «oligarquía» y por las capas medias cuya expansión había sido cohibida por la política de los gobiernos anteriores, planteaba el crecimiento económico y la integración nacional como metas primordiales. Dada su política explícita de crear una infraestructura de comunicaciones y transportes a nivel nacional y de racionalizar - hasta donde fuera posibleanticuados modos de producción, no sorprende que los primeros años del régimen vieran la promulgación de un cuerpo de leyes pro-indias, así como la creación de varias agencias burocráticas y tutelares, entre ellas una Sección de Asuntos Indígenas, dentro del Ministerio de Fomento, y el «Patronato de la Raza Indígena», tan denunciado por críticos como Dora Mayer.

Las mismas metas de crecimiento económico e integración nacional, al igual que la resistencia de los sectores conservadores, explican por qué la política indigenista de Leguía tuviera un carácter al parecer contradictorio. En las palabras de Alfajeme y Valderrama:

Se manifestaba un interés por impulsar el desarrollo del Perú como nación en términos de expansión del mercado interno y en términos de la consolidación de un aparato estatal más fuerte. La expansión del mercado interno, que buscaba una mayor incorporación de la población indígena a la economía como fuerza de trabajo y como pro-

${ }^{4}$ Augusta Alfajeme y Mariano Valderrama, «Viejas y nuevas fracciones dominantes frente al problema indígena: 1900-1930», en Indigenismo, p. 90. 
ductora y consumidora de mercancías, implicaba, en las condiciones concretas de nuestro país, la sobreexplotación de la mano de obra aborigen ${ }^{5}$.

Dentro del panorama global de la «sobreexplotación de la mano de obra aborigen» se destaca el programa de conscripción vial, instituido por el gobierno en 1921, y que constituyó un impuesto oneroso para las comunidades indígenas ( $\mathrm{y}$, sea dicho de paso, en nada se parecía a la gloriosa y casi sobrehumana faena de la construcción de la carretera Puquio-Nazca narrada por Arguedas en Yawar fiesta). Para 1923, el gobierno de Leguía ya había perdido el apoyo inicial de intelectuales indigenistas como Félix Cosío, Luis E. Valcárcel, Hildebrando Castro Pozo, José Antonio Encinas, Abelardo Solís y Erasmo Roca, muchos de los cuales se habían pasado a la oposición anti-reeleccionista. Después de 1923, la acción indigenista gubernamental se redujo a la implementación de reformas ya promulgadas y a una campaña manipulativa de retórica pro-india, que tuvo expresión cabal en el derecho de 1930 que estableció el 24 de junio como «Día del Indio».

Uno de los factores sociales que diferencia la década de los veinte de la anterior es la incorporación de la pequeña burguesía urbana al sistema político, constituyendo ella, sobre todo el sector empleados, uno de los pilares de apoyo al régimen leguiísta. Surge y se define, a la vez, una pequeña burguesía provinciana. Sectores importantes de esta nueva clase se radicalizan y derivan hacia posiciones anti-imperialistas y antileguiístas, y en el Sur, agresivamente regionalistas. Por estos años se constituyen, como es bien sabido, los núcleos directores de los partidos aprista y comunista, los primeros partidos de masas en el Perú, y se cuestiona tanto la hegemonía ejercida por el capital extranjero como la política desarrollista del gobierno central. Este es el caso, sobre todo en el Sur, región que, como viene demostrando Alberto Flores-Galindo, durante los dos siglos anteriores había formado un espacio económico bastante desligado del resto del país ${ }^{6}$. Es en el Sur donde, en las primeras décadas del siglo $\mathrm{xx}$, se presenta un nuevo regionalismo con «un fuerte acento antigamonalista e indigenista» ${ }^{7}$. Es un hecho que muchos

${ }^{5}$ Alfajeme y Valderrama, «Viejas y nuevas fracciones», p. 91.

- Alberto Flores-Galindo, Arequipa y el sur andino: ensayo de historia regional (siglos XVIII-XX) (Lima: Editorial Horizonte, 1977). Algunas de las conclusiones de Flores-Galindo han sido criticadas por Baltazar Caravedo en «Poder central y descentralización: Perú, 1931», Apuntes, 5, núm. 9 (Lima, 1979), pp. 111-129.

${ }^{7}$ Marfil Francke Ballve, «El movimiento indigenista en el Cuzco (1910-1930)», presentado originalmente como Memoria para optar al Grado de Bachiller de Cien- 
de los indigenistas peruanos más destacados del oncenio salieron de las filas de los abogados, médicos, pequeños comerciantes y maestros sureños, sobre todo en los departamentos de Puno (estudiado por Dan Hazen en una tesis doctoral magnífica) ${ }^{8}$ y del Cuzco. Son intelectuales de los sectores medios, que conforman, por ejemplo, el "Grupo Resurgimiento" en el Cuzco para fines de 1926. Habría que constatar también, sin embargo, una fuerte tendencia centrípeta que se manifiesta en la emigración de gran parte de la joven intelectualidad provinciana a la capital y en el hecho de que dos de las revistas «indigenistas» (en un sentido lato) de mayor circulación e influencia en los años veinte, Amauta y La Sierra, se editaran en Lima.

Como señala Flores-Galindo en otro estudio, al par que crecían y se diferenciaban los sectores medios urbanos a comienzos del siglo presente, se produjo un aumento dramático en el número de los lectores y en las aperturas de nuevas editoriales y librerías. Entre 1918 y 1928, el número de periódicos y revistas publicados en el país casi se triplica, subiendo de 167 a 473 . Muchos de los nuevos títulos tenían un carácter político e informativo ${ }^{9}$. Bruno Podestá, en un artículo reciente sobre revistas peruanas del siglo $\mathrm{xx}$, recalca la importancia de estas publicaciones «como conjunto expresivo del trabajo artístico y científico, como tribunas de la discusión teórica y metodológica, como expresión —radical algunas veces, conservadora otras- de la pequeña burguesía» ${ }^{10}$.

Los años veinte vieron brotar una cantidad impresionante de revistas «indigenistas» tanto en provincias como en Lima. Al igual que las publicaciones «vanguardistas» contemporáneas, estas revistas se caracterizaban por sus cortas tiradas y su vida generalmente breve, pero el crecido número de tales órganos manifiesta hasta qué punto el interés por lo indígena había captado la atención pública. A pesar de que revistas como Kosko, Attusparia, Kúntur, Chirapu, Inti, Puna y otras publicaran una parte ponderable de las contribuciones al debate en torno al "problema indígena», es sólo en años recientes cuando se las empieza a investigar sistemáticamente. Luis Enrique Tord, en un libro desigual, El indio en los ensayistas peruanos, 1848-1948, dedica poco espacio al

cias Sociales en la Universidad Católica del Perú, reproducido en Indigenismo, pp. 107-186. La cita proviene de la p. 111.

${ }^{8}$ Dan Chapin Hazen, «The Awakening of Puno: Government Policy and the Indian Problem in Southern Peru, 1900-1955», Ph. D. Diss. Yale Univ., 1974.

${ }^{y}$ Flores-Galindo, «Los intelectuales y el problema nacional», pp. 142-144.

${ }^{10}$ Bruno Podestá, «Revistas peruanas de este siglo», en Francisco Miró Quesada C. y otros, Historia, problema y promesa: homenaje a Jorge Basadre (Lima: Pontificia Universidad Católica del Perú, Fondo Editorial, 1978), II, 263. 
papel desempeñado por estas revistas dentro del debate nacional, y le corresponde a Marfil Francke Ballve, en una tesis universitaria ( $\mathrm{E} 1 \mathrm{mo}$ vimiento indigenista en el Cuzco [1910-1930]»), demostrar hasta qué punto un examen detallado de tales fuentes puede contribuir al estudio de la historia de las ideas en el Perú moderno ${ }^{11}$. Sin embargo, no existen todavía (exceptuamos aquí a Amauta) índices de las numerosas revistas indigenistas del oncenio ni mucho menos se ha reconstruido la compleja red de comunicaciones que ligaban a los indigenistas de provincias con los de la capital y con los de otros países de la América Latina. Creo que hacen mucha falta todavía estudios literarios de tipo «arqueológico» que permitan el manejo libre de estas fuentes de informaciones.

No cabe aquí hablar del conjunto de revistas «indigenistas» de la década de los años veinte, aunque fue en una de ellas, Inti, de Huancayo, donde el joven Arguedas publicara uno de sus primeros artículos ${ }^{12}$. Conviene sólo contrastar brevemente dos opciones políticas frente al problema agrario y campesino, que encontraron expresión en las revistas Amauta (Lima, 1926-1930), Kúntur (Cuzco, 1927-1928) y La Sierra (Lima, 1927-1930). Creo que Amauta ya está bastante conocida para no exigir un comentario detallado ${ }^{13}$. Aunque tal vez se recuerde con mayor frecuencia como "la primera revista socialista marxista en el Perú» ${ }^{14}$. Amauta, dirigida por José Carlos Mariátegui y luego por Ricardo Martínez de la Torre, sirvió también como uno de los principales foros indigenistas de la inteligencia radicalizada. La revista adoptó desde su creación, en 1926, un estilo artístico conscientemente indigenista (debido principalmente a la participación activa de José Sabogal), publicó grandes cantidades de poesía nativista y abordó desde múltiples perspectivas el problema agrario/campesino durante sus cuatro años de vida. Las

${ }^{11}$ Luis Enrique Tord, El indio en los ensayistas peruanos, 1848-1948 (Lima: Editoriales Unidas, 1978); Francke Ballve, obra citada. En el presente ensayo se siguen, en sus líneas generales, los argumentos de Francke Ballve.

${ }^{12}$ No hemos podido consultar este artículo del joven Arguedas, titulado «E1 presente y el pasado del indígena», y publicado en Inti (Huancayo) en 1928. Moisés Arroyo Posadas, «La multitud y el paisaje peruanos en los relatos de José María Arguedas», Cuadernos de crítica literaria y social (Lima, 1935), pp. 11-12, citado en A. Urrello, José María Arguedas: el nuevo rostro del indio (Lima: Juan Mejía Baca, 1974), p. 77.

${ }^{13}$ Para una discusión de las actitudes indigenistas manifestadas en la revista Amauta, véase David Wise, «A Peruvian Indigenista Forum of the 1920s: José Carlos Mariátegui's Amauta», Ideologies and Literature, 3, núm. 13 (junio-agosto 1980), pp. 70-104.

${ }^{14}$ Jorge Falcón, Amauta: polémica y acción de Mariátegui (Lima: Empresa Editora Amauta, 1979), p. 5. 
contribuciones de Mariátegui y de varios de sus colaboradores sobre los problemas nacional e indígena representan el primer intento de enfocar el "problema del indio» dentro de los planteamientos del socialismo científico. Como es bien sabido, Mariátegui llegó a otorgar al campesinado importancia decisiva en la instauración del socialismo en el Perú, llegando en su praxis (y en las páginas de su revista) a formulaciones políticas parecidas en ciertos aspectos a las desarrolladas por su contemporáneo Mao Tse-Tung ${ }^{15}$.

Secciones de Amauta, además, sobre todo la titulada «E1 proceso del gamonalismo», sección de denuncia antilatifundista, ponen de relieve la preocupación de Mariátegui y sus colegas por los abusos a los cuales el campesinado seguía sometido dentro de la «Patria Nueva» modernizadora de Leguía. Conviene recordar el testimonio de José María Arguedas - uno de muchos escritores de los años treinta que recibieron el impacto profundo de los escritos de Mariátegui:

Fue leyendo a Mariátegui y después a Lenin que encontré un orden permanente en las cosas; la teoría socialista no sólo dio un cauce a todo el porvenir, sino a lo que había en mí de energía, le dio un destino y lo cargó aún más de fuerza por el mismo hecho de encauzarlo ${ }^{16}$.

Cabe mencionar, sin embargo, que tanto en su indigenismo como en sus planteamientos en torno al arte de vanguardia, Amauta deja notar el bien conocido eclecticismo editorial de Mariátegui. Dada la heterogeneidad de los autores que escribían para Amauta, no sorprende la persistencia de una corriente indigenista rapsódica y telúrica, ni la presencia de artículos que versan sobre reformas esencialmente pedagógicas, ni la coexistencia de opiniones discrepantes en torno al «socialismo incaico». Vale destacar, al comentar Amauta, la posición prominente otorgada a los ensayos lírico-mesiánicos de Luis E. Valcárcel, provenientes del libro Tempestad en los Andes, de 1927, así como la publicidad prestada al "Grupo Resurgimiento», un frente de intelectuales cuzqueños sofocado por la represión gubernamental en 1927, a pocos meses de su creación.

${ }^{15}$ Véase Harry E. Vanden, «The Peasants as a Revolutionary Class: An Early Latin American View», Journal of Interamerican Studies and World Affairs, 20, núm. 2 (mayo 1978), 191-209.

${ }^{16}$ José María Arguedas, El zorro de arriba y el zorro de abajo (Buenos Aires: Losada, 1971), pp. 297-298. Escajadillo (pp. 101-102) afirma como «evidente» el hecho de que la obra narrativa de Arguedas está marcada por la influencia de los 7 ensayos de Mariátegui y de Amauta. 
Dentro del «ala izquierda» del indigenismo peruano, una revista ideológicamente más homogénea, pero de vida brevísima, fue Kúntur (Cóndor), publicada en el Cuzco en 1927 y 1928 por una fracción de la entonces célula aprista cuzqueña. Muchos de los colaboradores de esta revista provinieron de la Universidad Popular González Prada en el Cuzco, del grupo estudiantil «Ande» y de la revista precursora Kosko (1924-1925). Varios de los redactores y colaboradores de Kúntur conformaron después el Partido Comunista en el Cuzco, independientes del «Grupo de Lima», encabezado por Mariátegui. Como para Mariátegui, para el grupo editor de Kúntur «el problema indígena era fundamentalmente de orden económico y político: sus raíces estaban en el régimen del latifundio y la dominación gamonalista... Solamente mediante una revolución popular, de carácter socialista, se podría dar fin a esa situación» ${ }^{17}$. En suma, sólo la alianza de los obreros y campesinos con el estudiantado radical podría llevar a cabo la lucha revolucionaria, que suprimiría los latifundios e implantaría el sovietismo agrario. Los colaboradores de Kúntur atacaron duramente no sólo el gamonalismo y el «indigenismo oficial» del gobierno de Leguía, sino también a indigenistas menos radicales (es el caso de Luis E. Valcárcel), y sobre todo la clase de indigenismo representado por la revista La Sierra. A causa de su agresividad política, Kúntur duró poco, siendo clausurada después de la aparición de su segundo número, en enero de 1928.

Mientras muchos intelectuales de provincias evolucionaron hasta posiciones políticas radicales, otros adoptaron actitudes más equívocas. La revista La Sierra, dirigida en Lima por J. Guillermo Guevara entre 1927 y 1930, representa lo que se podría denominar el «ala derecha» del indigenismo que encontraba expresión en las pequeñas revistas de la década. Levantó estridentemente banderas «serranistas» o «andinistas» a la vez que dejaba percibir un racismo disfrazado por un paternalismo «humanista». Ocupan una posición preeminente en La Sierra las doctrinas «indolatinas» y "supranacionales» de Víctor J. Guevara, catedrático de Jurisprudencia en la Universidad del Cuzco. También son de notar las colaboraciones en La Sierra del hacendado progresivo Rafael Larco Herrera, dueño de la hacienda azucarera «Chiclín», en el valle del Chicama (de la costa norte). Atacó La Sierra, es cierto, el gamonalismo "retrógrado" de la sierra, planteando la solución al problema agrario en términos de la modernización capitalista. Las posiciones indigenistas de los Guevara, a fin de cuentas, se parecían bastante a las

\footnotetext{
${ }^{17}$ Francke Ballve, p. 154.
} 
adoptadas por el Partido Aprista Peruano en su Programa Mínimo de 1931, al reclamar la modernización y tecnificación de la agricultura, la cooperativización de las comunidades indígenas y el fomento de la pequeña propiedad, al par de la «incorporación del indio a la vida nacional» por medio de la enseñanza y de una vigorosa campaña contra el uso del alcohol y de la coca.

Creo que lo que hace de La Sierra una revista de la «derecha» no es tanto su paternalismo patente como su racismo no siempre bien solapado. Según declaraba Víctor J. Guevara, el indígena peruano se encontraba en un «estado de atraso y cuasi degeneración», adicto al alcohol y refractario a la higiene y a la enseñanza moral. Para solucionar el problema más grave -el alcoholismo-, sugirió Víctor Guevara la adopción de una «ley seca» del tipo que, según él, tan buenos resultados había dado en los Estados Unidos y en Rusia ${ }^{18}$. Y en cuanto al problema de integrar al campesino indígena al mercado interno del país, convirtiéndolo en consumidor, escribió:

La acción de las leyes puede en este caso resultar de alguna eficacia. ¿Por qué la ley no podría prescribir la forma y la calidad de sus ropas? ¿Por qué no los compartimentos y las condiciones higiénicas de sus viviendas? ¿Por qué no ciertos usos y espectáculos? ${ }^{19}$.

Sin embargo, según Guevara, la acción legislativa en sí, por vigorosa que fuera, no bastaba para eliminar las taras biológicas del indígena, de modo que

en atención a que el temperamento de la raza es servil, apático, holgazán, supersticioso, retardatario, conviene cruzarla con los pueblos de las razas europeas dotadas de las cualidades contrarias a esos defectos, capaces de producir un equilibrio progresivo ${ }^{20}$.

Nos encontramos ahora, por supuesto, en el campo del biologismo abierto. La propuesta eugenésica de Víctor Guevara recibiría de parte de los marxistas de Kúntur el mote burlón de «solución bovina». En con-

${ }^{18}$ Véase Saúl de Navarro, «'Hacia Indolatinia', de Víctor J. Guevara», La Sierra, año I, núm. 7 (julio 1927), p. 39. Navarro se refiere al libro Hacia Indolatinia de Víctor J. Guevara, publicado en el Cuzco en 1926.

19 Víctor J. Guevara, "La reforma del indio», La Sierra, año I, núm. 1 (enero 1927), p. 8. Citado también por Francke Ballve, p. 149.

${ }^{20}$ Víctor J. Guevara, «El problema del indio», La Sierra, año I, núm. 2 (febrero 1927), p. 10. Citado también por Francke Ballve, p. 150. 
traste, J. Guillermo Guevara, director de La Sierra, opinaba que el aborigen peruano no era un

especimen humano que marcha hacia la completa decadencia, es más bien un factor redimible, que conserva incólume el espíritu de la raza, accesible a su adaptación en las formas modernas de la vida ${ }^{21}$.

Lo cual, como ya se ha indicado, implicaba la incorporación del campesino al proceso del desarrollo capitalista nacional.

Tal vez sea erróneo plantear una polaridad absoluta entre La Sierra y su contemporánea Amauta, ya que varios indigenistas de peso - entre ellos Luis Valcárcel y Emilio Romero- colaboraron en ambas revistas, como lo hizo también un destacado intelectual de la llamada «generación de 1919», Jorge Basadre. Publicó La Sierra, además, en uno de sus últimos números, la respuesta de Mariátegui a un cuestionario preparado por un «Seminario de Cultura Peruana» sobre el problema agrario nacional $^{22}$. Cabe añadir también que la retórica antigamonalista de La Sierra resultaba menos tímida al surgir dentro de la fase represiva del régimen leguiísta de lo que parecería en décadas posteriores.

En lo que sí se diferencia La Sierra de publicaciones como Amauta y Kúntur son su rechazo enfático al socialismo como doctrina «ajena», su antilimeñismo agresivo y su énfasis en la pedagogía como medida adecuada a solucionar la marginalidad del indígena y de «humanizarlo». La revista sirvió también como plataforma para la doctrina de la «supranacionalización de la prensa», doctrina peregrina y pacifista de Víctor J. Guevara, que solucionaría los conflictos bélicos del continente sudamericano y del mundo mediante la creación de un organismo supranacional de periodistas dotados de inmunidad política. Igualmente salta a la vista la discrepancia entre los héroes intelectuales y políticos adoptados por Amauta y por La Sierra: en aquélla, los líderes de la Revolución rusa y de la creación del nuevo Estado soviético - Lenin, Trotski, Lunatcharski-; en ésta, los voceros de un americanismo telúrico y antiimperialista -Manuel Ugarte, Franz Tamayo, Ricardo Rojas, Alfredo L. Palacios y Víctor Raúl Haya de la Torre-. Claro que en los culture heroes de las dos revistas existían ciertas coincidencias notables, como lo demuestran los casos de José Vasconcelos, de Henri Barbusse y del líder guerrillero Sandino.

${ }^{21}$ J. Guillermo Guevara, «Editorial», La Sierra, año I, núm. 1 (enero 1927), p. 2. Citado también por Francke Ballve, p. 149.

22 José Carlos Mariátegui, «Respuesta al cuestionario No. 4 del "'S. de C. P.'», La Sierra, año III, núm. 29 (1929), pp. 56-60. 
Como Amauta, como la mayoría de las publicaciones periódicas peruanas, La Sierra naufraga en la crisis económica y política que se desató en 1930. Es en este momento donde, ante el colapso económico generalizado y la lucha por el poder entre la oligarquía tradicional por una parte y el APRA, convertido en partido de masas, por otra, el indigenismo político retrocede. Como nos indica Carlos Iván Degregori, sobrevive, debilitado, en la retórica aprista, en la literatura, en los estudios antropológicos y folklóricos, en la Escuela de Bellas Artes, dirigida por José Sabogal ${ }^{23}$. Será, sin embargo, por lo menos a corto plazo, un movimiento políticamente innocuo, aunque bien de intensa resonancia en el campo artístico.

${ }^{23}$ Carlos Iván Degregori, «Ocaso y replanteamiento de la discusión del problema (1930-1977)», en Indigenismo, p. 235. 
